Ferrata Storti Foundation

\title{
Fecal microbiota transplantation before or after allogeneic hematopoietic transplantation in patients with hematologic malignancies carrying multidrug-resistance bacteria
}

Haematologica 2019

Volume 104(8):1682-1688

\section{Correspondence: \\ GIORGIA BATTIPAGLIA \\ giorgia.battipaglia@aphp.fr \\ Received: May 29, 2018. \\ Accepted: January 31, 2019. \\ Pre-published: February 7, 2019.}

doi:10.3324/haematol.2018.198549

Check the online version for the most updated information on this article, online supplements, and information on authorship \& disclosures: www.haematologica.org/content/104/8/1682

(C)2019 Ferrata Storti Foundation

Material published in Haematologica is covered by copyright. All rights are reserved to the Ferrata Storti Foundation. Use of published material is allowed under the following terms and conditions:

https://creativecommons.org/licenses/by-nc/4.0/legalcode. Copies of published material are allowed for personal or internal use. Sharing published material for non-commercial purposes is subject to the following conditions:

https://creativecommons.org/licenses/by-nc/4.0/legalcode, sect. 3. Reproducing and sharing published material for commercial purposes is not allowed without permission in writing from the publisher.

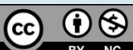

\author{
Giorgia Battipagliaa, ${ }^{1,2}$ Florent Malard, ${ }^{1,3}$ Marie Therèse Rubio, ${ }^{1,4,5}$ \\ Annalisa Ruggeri, ${ }^{1}$ Anne Claire Mamez, ${ }^{1}$ Eolia Brissot, ${ }^{1,3}$ Federica Giannotti, ${ }^{1}$ \\ Remy Dulery, ${ }^{1}$ Anne Christine Joly, ${ }^{6}$ Minh Tam Baylatry, ${ }^{6}$ \\ Marie Jeanne Kossmann, ${ }^{7}$ Jacques Tankovic, ${ }^{8}$ Laurent Beaugerie, ${ }^{6,9}$ \\ Harry Sokol and ${ }^{3,9,10,11^{\star}}$ Mohamad Mohty ${ }^{1,3^{*}}$
}

\begin{abstract}
*These authors contributed equally to this work as co-senior authors
${ }^{1}$ Department of Hematology, Saint Antoine Hospital, Paris, France; ${ }^{2}$ Federico II University, Hematology Department, Naples, Italy; ${ }^{3}$ Sorbonne Universités, UPMC Univ Paris 06, INSERM, Centre de Recherche Saint-Antoine (CRSA), F-75012 Paris, France; ${ }^{4}$ Service d'Hématologie, Hôpital Brabois, CHRU Nancy, France; ${ }^{5}$ CMRS UMR 7563, IMoPa, Biopole de I'Université de Lorraine, France; ${ }^{6}$ Microbiote Transplant Préparations Unit, Pharmacy Department, Saint Antoine Hospital, Paris, France; 'Unité d'Hygiène et de Lutte Contre les Infections Nosocomiales, Saint Antoine Hospital, Paris, France; ${ }^{8}$ Department of Bacteriology, Saint Antoine Hospital, Paris, France; ' $D$ Department of Gastroenterology, Saint Antoine Hospital, AP-HP, Paris, France; ${ }^{10}$ Sorbonne Université, École Normale Supérieure, PSL Research University, CNRS, INSERM, AP-HP, Hôpital Saint-Antoine, Laboratoire de Biomolécules, LBM, F-75005 Paris, France and ${ }^{11}$ INRA, UMR1319 Micalis \& AgroParisTech, Jouy en Josas, France
\end{abstract}

\section{ABSTRACT}

Tecal microbiota transplantation is an effective treatment in recurrent -1 Clostridium difficile infection. Promising results to eradicate multidrugresistant bacteria have also been reported with this procedure, but there are safety concerns in immunocompromised patients. We report results in ten adult patients colonized with multidrug-resistant bacteria, undergoing fecal microbiota transplantation before $(n=4)$ or after $(n=6)$ allogeneic hematopoietic stem cell transplantation for hematologic malignancies. Stools were obtained from healthy related or unrelated donors. Fecal material was delivered either by enema or via nasogastric tube. Patients were colonized or had infections from either carbapenemase-producing bacteria $(n=8)$ or vancomycin-resistant enterococci $(n=2)$. Median age at fecal microbiota transplantation was 48 (range, 16-64) years. Three patients needed a second transplant from the same donor due to initial failure of the procedure. With a median follow up of 13 (range, 4-40) months, decolonization was achieved in seven of ten patients. In all patients, fecal microbiota transplantation was safe: one patient presented with constipation during the first five days after FMT and two patients had grade I diarrhea. One case of gut grade III acute graft-versus-host disease occurred after fecal microbiota transplantation. In patients carrying or infected by multidrugresistant bacteria, fecal microbiota transplantation is an effective and safe decolonization strategy, even in those with hematologic malignancies undergoing hematopoietic stem cell transplantation.

\section{Introduction}

During the last decades, the prevalence of multidrug-resistant bacteria (MDRB) has largely increased, becoming a serious worldwide problem. ${ }^{1}$ Under physiological conditions, commensal microbiota prevents gut colonization from MDRB. However, in particular conditions, such as in patients with hematologic malignancies, use of chemotherapeutic agents and broad spectrum antibiotics may favor selection of resistant pathogens through the alterations of the gastrointestinal barrier and the consequent dysbiosis. ${ }^{2}$ Patients undergoing allogeneic hematopoietic stem cell transplantation (allo-HSCT) are at even higher risk of dysbiosis due to 
their profound immune depression. ${ }^{3}$ In cases of bloodstream infections from MDRB, outcomes are even poorer, leading to increased mortality. ${ }^{4}$ For example, an Italian study showed that carbapenemase producing (CP-) bacteria, including Pseudomonas aeruginosa, were independent predictors of death in patients diagnosed with acute leukemia, while this was not observed in cases of extended-spectrum $\beta$-lactamase (ESBL) Enterobacteriaceae. ${ }^{5}$

In order to prevent bacteria spreading to other patients, preventive measures are required, including isolating patients, limitating transfer to other healthcare centers, and management by dedicated staff. These measures result in an increase in healthcare costs, which cannot always be met. ${ }^{6}$ According to French recommendations, patients colonized with MDRB may be denied access to healthcare facilities if dedicated staff are not available. ${ }^{7}$

New classes of antibiotics are under study to treat infections related to $M D R B$, and research is ongoing to find effective decolonization strategies. ${ }^{8}$ The use of oral gentamicin was initially proposed in some MDR-gram negative strains but failure is common, and management of gentamicin-resistant strains may also be an issue. , $^{10}$

Fecal microbiota transplantation (FMT) is a procedure that has been proved to be effective and safe in the treatment of recurrent Clostridium difficile infection (CDI), and it is now a recommended therapy in this setting. ${ }^{11}$ Use of FMT in patients carrying MDRB is still at an investigational stage, but there are reports and case series showing its efficacy in this setting. ${ }^{12,13}$ Many concerns were initially raised about the feasibility of FMT in immunocompromised patients, such as those affected by hematologic malignancies, because of the theoretical potential for local and bloodstream infections. However, recent case reports revealed the efficacy and safety in this particular population. ${ }^{14-16}$ Recently, Bilinski et al. reported the results of a prospective study evaluating FMT in 20 patients with MDRB gut colonization and contemporarily affected by hematologic malignancies. Overall 25 FMT were performed and 15 of 20 patients experienced complete MDRB decolonization, ${ }^{17}$ including some of them with graft-versus-host disease (GvHD) after allo-HSCT.

In this retrospective study, we report our experience with FMT in patients diagnosed with hematologic malignancies and undergoing FMT either before or after alloHSCT.

\section{Methods}

In this single-center study, we retrospectively analyzed data on all consecutive adult patients diagnosed with hematologic malignancies who underwent FMT before or after allo-HSCT due to MDRB colonization. In our center, microbiological screening is performed weekly in all inpatients, with consequent preventive measures in positive patients in order to limit MDRB spread, according to national guidelines. ${ }^{7}$ (See Online Supplementary Appendix for details).

This study was approved by the institutional ethics committees. The treatment plan was discussed in advance by a multidisciplinary team (hematologist, gastroenterologist, pharmacist) who approved the procedure. The decision was made on a patient-topatient basis. All patients signed informed consent explaining the theoretical risks of the procedure because of the current investigational use of FMT in the field of MDRB and in patients with hematologic malignancies.
Eligibility criteria included: asymptomatic carriers or systemic infections from vancomycin-resistant enterococci (VRE), carbapenemase-producing Enterobacteriaceae (CPE) or CP-Pseudomonas aeruginosa. The rationale for FMT and MDRB decolonization were mainly to limit infectious complications related to these bacteria and to facilitate patient transfer to other departments, such as intensive care units or rehabilitation centers.

Contemporary colonization from ESBL-producing bacteria was also registered in patients undergoing FMT. We, therefore, subsequently evaluated whether FMT also allowed decolonization from these MDRB.

For the purpose of this retrospective analysis, we also classified $\mathrm{MDRB}$ as multi-drug (MDR), extensive-drug (XDR) and pan-drugresistant (PDR), according to the definition proposed by Magiorakos et al. ${ }^{18}$ MDR was defined as the presence of acquired non-susceptibility to at least one agent in three or more antimicrobial categories, XDR as non-susceptibility to at least one agent in all but two or fewer antimicrobial categories (i.e. bacterial isolates remain susceptible to only one or two categories), and PDR as non-susceptibility to all agents in all antimicrobial categories. Details on donor selection, microbiological testing, fecal material preparation and delivery are available in the Online Supplementary Appendix.

Decolonization from VRE, CPE or CP-Pseudomonas aeruginosa after negative results on a minimum of three consecutive microbiological cultures (performed weekly) was defined as "major decolonization", while "persistent decolonization" was defined as the persistence of negative rectal swab until last follow up after a first or second FMT, whenever this was feasible. In patients concomitantly colonized by ESBL-producing Enterobacteriaceae, "concomitant decolonization" was defined as negative results on at least three consecutive rectal swabs after FMT. The safety of the procedure was also registered. For all patients, data on significant infections (defined as bacteriemia or sepsis occurring during the first 90 days after FMT) were also collected. Although febrile neutropenia or fever of unknown origin was not considered a significant infectious episode, such events were also recorded. A second attempt could be proposed in those patients presenting either a relapse of MDRB colonization or experiencing FMT failure.

\section{Results}

During the period between 2014 and 2017, ten patients underwent FMT: seven due to gut colonization without systemic infection by either CPE (Escherichia coli, $\mathrm{n}=1$; Citrobacter freundii, $\mathrm{n}=2$; Klebsiella pneumoniae, $\mathrm{n}=1$ ), or $C P$ Pseudomonas aeruginosa $(\mathrm{n}=1)$ or VRE $(\mathrm{n}=2)$, and three after having experienced systemic infections from $C P$ Pseudomonas aeruginosa. Median age at FMT was 48 (range, 16-64) years. Four patients underwent FMT as a decolonization strategy before allo-HSCT, with a median interval from FMT to transplant of 28 (range, 9-46) days. Of note, one patient was contemporarily colonized by three different CPE. Two patients started conditioning regimen three days after FMT and the other two after a month. Six patients underwent FMT after allo-HSCT, with a median time from allo-HSCT to FMT of 163 (range, 98-344) days. Of note, all patients undergoing FMT after allo-HSCT were still on immunosuppressive therapy at the time of FMT, with only one of six presenting active grade IV steroid-dependent gut GvHD. Overall, six patients were also colonized by ESBL-producing Enterobacteriaceae. All ESBL-producing bacteria were classified as MDR.

A frozen product was used in eight of ten patients and 


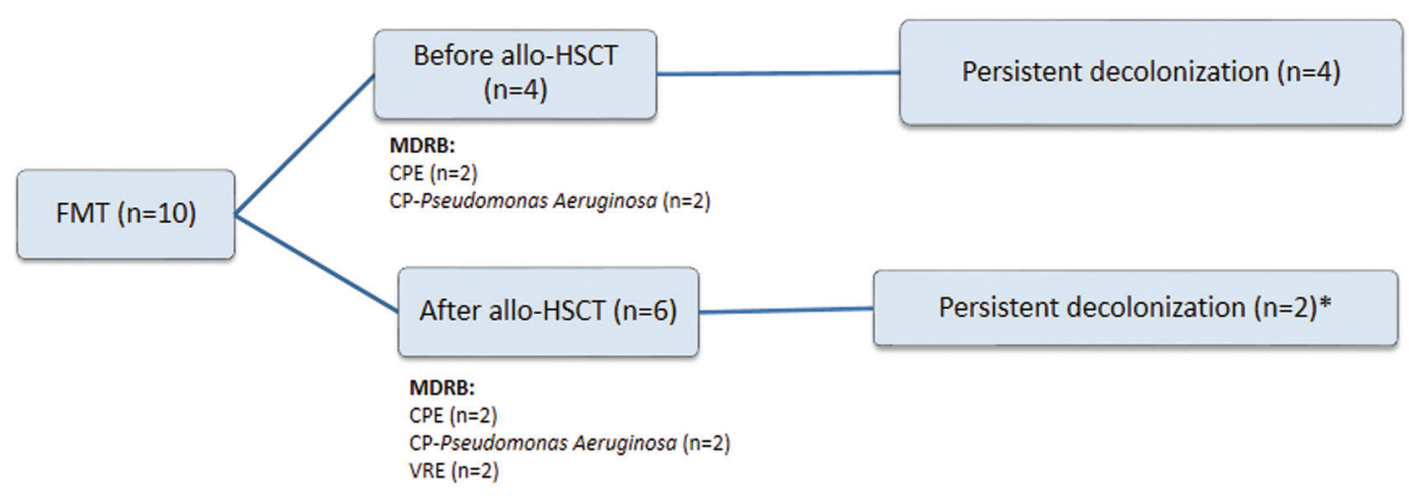

Figure 1. Results of fecal microbiota transplantation. FMT: fecal microbiota transplantation; allo-HSCT: allogeneic hematopoietic stem cell transplantation; CPE: carbapenemase-producing Enterobacteriaceae; CP-Pseudomonas Aeruginosa: carbapenemase producing Pseudomonas Aeruginosa; VRE: vancomycin resistant enterococci. *A third patient achieved decolonization from vancomycin-resistant enterococci and then experienced recurrence of colonization 20 months after fecal microbiota transplantation, concomitantly to disease relapse.

enema was the preferred method of administration in all but one patient who presented a compromised neurological status due to a cerebral toxoplasmosis and was not considered eligible for enema. Median donor stool quantity was $84 \mathrm{~g}$ (range, 43-104 g). At the time of FMT, neutrophil count was $>1 \times 10^{9} / \mathrm{L}$ in all patients but one who had a neutrophil count of $0.17 \times 10^{9} / \mathrm{L}$ (with steroid-resistant GvHD). Platelet count was count $>20 \times 10^{9} / \mathrm{L}$ in all patients.

Three patients required a second FMT. In one patient, after initial efficacy, VRE was again detectable two months after the first FMT. This patient developed multiple infectious episodes (particularly sinusitis and pneumonia), prompting the frequent use of large spectrum antibiotics; this probably led to recurrence of VRE colonization. In the other two patients, a second attempt was made due to the failure of the first procedure. In one patient, failure was mainly attributable to incorrect preparation with PEG (insufficient intake). After a second attempt with a correct preparation, VRE eradication was achieved and maintained until 20 months after FMT, at which point, VRE was detectable at the same time as recurrence of hematologic disease. In the last patient, administration of first and second FMT was mainly for compassionate use to treat active grade IV gut GvHD and multiple infectious episodes which made withdrawal of antibiotics impossible, even during the 72 hours (h) following FMT (see below).

Globally, major decolonization (three consecutive negative microbiological cultures) was achieved in seven of ten patients, including two patients after a second FMT (Figure 1). Persistent decolonization (negative microbiological cultures at last follow up) was achieved in six of ten patients after a median follow up of 13 months (range, 4-40 months) from FMT. Indeed, as already mentioned, one patient presented a positive rectal swab for ERV 20 months after FMT contemporary to disease relapse. She later died due to hematologic progression.

Failure occurred in the remaining three patients. The patient undergoing FMT for compassionate use had presented multiple infectious episodes from CP-Pseudomonas aeruginosa, making it impossible to stop antibiotics during the $72 \mathrm{~h}$ after FMT. Moreover, grade IV gut GvHD was associated with intestinal occlusion, requiring aspiration via a nasogastric tube, at time of FMT. Despite two attempts with FMT, the procedure was a failure and the patient later died. In the second patient, due to the problems encountered in the positioning of a nasogastric tube, FMT was administered by enema and the patient was not able to retain the product for the advised 2-3 h; she refused a second attempt. The third patient underwent FMT by enema from an unrelated donor and the reason given for FMT failure was that she had not received sufficient stool quantity (43 g); however, this is not logical given that decolonization from concomitant ESBL-producing Enterobacteriaceae had been achieved. A second attempt in this patient was not possible due to the unavailability of additional material.

Among the six patients concomitantly colonized from ESBL-producing Enterobacteriaceae, three obtained concomitant decolonization.

Details on FMT performed before or after allo-HSCT are reported in Table 1. As an example of successful FMT, Figure 2 shows the case of the patient undergoing FMT from nasogastric tube, after experiencing breakthrough infectious episodes related to colonization from $C P$-Pseudomonas aeruginosa requiring continuous hospitalization for the first year after allo-HSCT. After FMT, this patient did not experience any other infectious episode and could finally be cared for as an outpatient.

With regards to the safety of FMT procedure, one patient presented constipation during the first five days after FMT which was favorably resolved after the use of laxatives, while two patients presented grade I diarrhea the day after FMT. No other major adverse events were observed.

Only one patient undergoing FMT before allo-HSCT developed a grade III acute gut GvHD at day +30 after allo-HSCT and at day +51 after FMT. A differential diagnosis with CMV colitis was made and she responded favorably to both antiviral and steroid treatment.

When looking at severe infectious episodes during the 90 days following FMT, in two of those patients undergoing FMT before allo-HSCT, documented bacteriemia without sepsis occurred early after allo-HSCT; these responded favorably to the introduction of large-spectrum antibiotics. In particular, one patient experienced a documented bacteriemia from multi-sensitive Pseudomonas 
aeruginosa at day +80 after allo-HSCT while the other patient experienced a documented bacteriemia from an ESBL-producing Escherichia Coli at day 60 after allo-HSCT. The additional two patients undergoing FMT before alloHSCT also received a large spectrum antibiotic such as piperacillin-tazobactam or cephalosporins for febrile neutropenia without documentation. Interestingly, despite the use of large spectrum antibiotics, no cases of MDRB recurrence were observed in these four patients.

Fungal and viral infections were observed in only one patient more than six months after FMT, but these were not considered to be related to FMT because this patient was under systemic immunosuppressive treatments for a cortico-resistant extensive GvHD (lung, skin, mucosal)

Table 1. Characteristics of patients undergoing fecal microbiota transplantation before (A) or after (B) hematopoietic stem cell transplantation.

\begin{tabular}{|c|c|c|c|c|c|c|}
\hline A & & 1 & \multicolumn{2}{|c|}{2} & 3 & 4 \\
\hline Patient sex & \multicolumn{2}{|r|}{ M } & \multicolumn{2}{|c|}{ M } & $\mathrm{F}$ & M \\
\hline Age at time of FMT, years & \multicolumn{2}{|r|}{64} & \multicolumn{2}{|c|}{42} & 45 & 47 \\
\hline \multicolumn{7}{|l|}{ B } \\
\hline Hematologic malignancy & \multicolumn{2}{|r|}{ AML } & \multicolumn{2}{|c|}{ AML } & AML & BPDCN \\
\hline Identified MDRB & \multicolumn{2}{|c|}{ CP-Pseudomonas aeruginosa } & \multicolumn{2}{|c|}{ CP-Pseudomonas aeruginosa } & $\mathrm{CPE}$ & $\mathrm{CPE}^{\circ}$ \\
\hline Antimicrobial resistance category & \multicolumn{2}{|c|}{ XDR } & \multicolumn{2}{|c|}{ MDR } & MDR & MDR \\
\hline Concomitant MDR-ESBL-producing bacteria colonization, & \multicolumn{2}{|c|}{ on, bacteria } & \multicolumn{2}{|c|}{$\mathrm{N}$} & Y & $\mathrm{N}$ \\
\hline Systemic infections due to MDRB before FMT & \multicolumn{2}{|r|}{ Y } & \multicolumn{2}{|c|}{$\mathrm{N}$} & $\mathrm{N}$ & $\mathrm{N}$ \\
\hline Time from FMT to allo-HSCT (days) & \multicolumn{2}{|r|}{41} & \multicolumn{2}{|c|}{46} & 16 & 9 \\
\hline FMT donor & \multicolumn{2}{|r|}{ Daughter } & \multicolumn{2}{|c|}{ Sister } & Husband & Sister \\
\hline Way of administration & \multicolumn{2}{|r|}{ Enema } & \multicolumn{2}{|c|}{ Enema } & Enema & Enema \\
\hline Major decolonization & \multicolumn{2}{|r|}{ Y } & \multicolumn{2}{|c|}{ Y } & Y & Y \\
\hline Persistent decolonization & \multicolumn{2}{|r|}{ Y } & \multicolumn{2}{|c|}{ Y } & Y & Y \\
\hline Concomitant ESBL-producing bacteria decolonization & & $\mathrm{Y}$ & & & $\mathrm{N}$ & $\mathrm{N} / \mathrm{A}$ \\
\hline Follow up after FMT, days & & 820 & & & 148 & 399 \\
\hline Follow up after allo-HSCT, days & & 779 & & & 132 & 390 \\
\hline Status & & Alive & & & Alive & Alive \\
\hline Cause of death & & N/A & Disease $p$ & ression & N/A & N/A \\
\hline Patient sex & $\mathrm{F}$ & M & $\mathrm{F}$ & $\mathrm{F}$ & $\mathrm{F}$ & $\mathrm{F}$ \\
\hline Age at time of FMT, years & 50 & 54 & 16 & 19 & 62 & 54 \\
\hline Hematologic malignancy & MPN & MPN & AML & ALL & MPN & ALL \\
\hline Identified MDRB & $\begin{array}{c}\text { CP-Pseudomonas } \\
\text { aeruginosa }\end{array}$ & $\begin{array}{c}\text { CP-Pseudomonas } \\
\text { aeruginosa }\end{array}$ & VRE & $\begin{array}{l}\text { VRE } \\
\text { CPE }\end{array}$ & CPE & \\
\hline Antimicrobial resistance category & PDR & XDR & XDR & XDR & MDR & XDR \\
\hline Concomitant MDR-ESBL-producing bacteria colonization & $\mathrm{N}$ & Y & Y & $\mathrm{Y}$ & $\mathrm{N}$ & Y \\
\hline Systemic infections due to MDRB before FMT & Y & $\mathrm{Y}$ & $\mathrm{N}$ & $\mathrm{N}$ & $\mathrm{N}$ & $\mathrm{N}$ \\
\hline Time from allo-HSCT to FMT & 324 & 344 & 98 & 160 & 123 & 167 \\
\hline FMT donor & Husband & Unrelated & Mother & Mother & Brother & Unrelated \\
\hline Way of administration & Nasogastric tube & Nasogastric tube & Enema & Enema & Enema & Enema \\
\hline Second FMT & $\mathrm{N}$ & Y & Y & Y & $\mathrm{N}$ & $\mathrm{N}$ \\
\hline Time from first to second FMT, days & N/A & 27 & 118 & 84 & N/A & N/A \\
\hline Major decolonization & Y & $\mathrm{N}$ & Y & Y & $\mathrm{N}$ & $\mathrm{N}$ \\
\hline Persistent decolonization & Y & N/A & Y & $\mathrm{N}$ & $\mathrm{N} / \mathrm{A}$ & N/A \\
\hline Concomitant ESBL-producing bacteria decolonization & N/A & $\mathrm{N}$ & $\mathrm{N}$ & Y & N/A & Y \\
\hline Colonization relapse & $\mathrm{N}$ & N/A & $\mathrm{N}$ & Y & $\mathrm{N} / \mathrm{A}$ & $\mathrm{N} / \mathrm{A}$ \\
\hline Follow-up after FMT, days & 678 & 33 & 1220 & 595 & 184 & 307 \\
\hline Follow-up after allo-HSCT, days & 1002 & 404 & 1436 & 839 & 307 & 474 \\
\hline Status & Alive & Dead & Alive & Dead & Alive & Alive \\
\hline Cause of death & $\mathrm{N} / \mathrm{A}$ & $\begin{array}{c}\text { Uncontrolled } \\
\text { GvHD and infection }\end{array}$ & N/A & $\begin{array}{c}\text { Disease } \\
\text { progression }\end{array}$ & N/A & N/A \\
\hline
\end{tabular}

${ }^{\circ}$ Three different types: Citrobacter freundii, Klebsiella Pneumoniae, Enterobacter Cloacae. F: female; M: male; FMT: fecal microbiota transplantation; AML: acute myeloid leukemia; BPDCN: blastic plasmacytoid dendritic cell neoplasm; MDRB: multidrug-resistant bacteria; CP: carbapenemase-producing; CPE: carbapenemase-producing Enterobacteriaceae XDR: extensively-drug resistant; MDR: multi-drug resistant; ESBL: extended-spectrum $\beta$-lactamase; Y: yes, N: no; allo-HSCT: allogeneic hematopoietic stem cell transplantation; GvHD: graft-versus-host disease; N/A: not applicable; MPN: myeloproliferative neoplasm; ALL: acute lymphoblastic leukemia;VRE: vancomycin-resistant enterococci; PDR: pandrug resistant. 


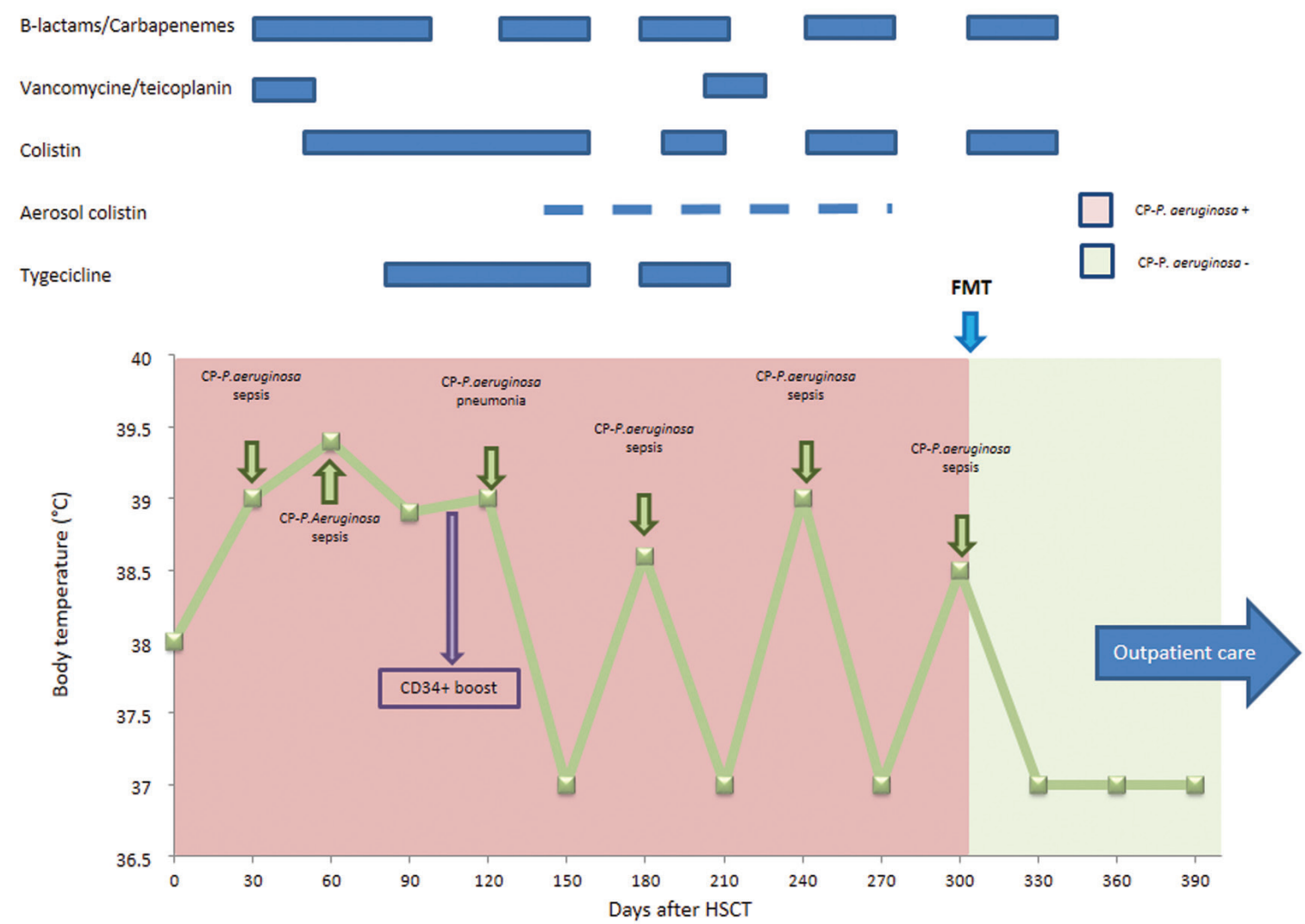

Figure 2. Evolution of Patient n. 5 as model of successful fecal microbiota transplantation. CP: carbapenemase-producing; FMT: fecal microbiota transplantation; HSCT: hematopoietic stem cell transplantation.

and infectious episodes exacerbated during immunosuppressive treatment. None of the other patients presented fungal or viral infections.

\section{Discussion}

The increasing emergence and diffusion of MDRB represents a major public health problem, with higher mortality in patients experiencing infections. This involves high costs of prolonged in-hospital care and preventive measures used to limit diffusion to other patients. ${ }^{6,19}$

Human gut microbiota, also named as "gut resistome", is the primary site for MDRB acquisition and colonization, being an important reservoir of antibiotic resistant genes. ${ }^{20}$ Patients diagnosed with hematologic malignancies are at high risk of colonization from MDRB. In fact, conditioning regimens for allo-HSCT and intensive chemotherapy significantly alter the gastrointestinal barrier and this modifies the composition of intestinal microbiota. Moreover, patients affected by hematologic malignancies or undergoing allo-HSCT are at particular risk for MDRB colonization or infection due to the large, prolonged and, sometimes, improper use of large spectrum antibiotics. ${ }^{2}$ Most bloodstream infections in hematologic patients derive from the gut, and infections are even more severe in those patients undergoing allo-HSCT, with high mortality rates of $36-95 \% .{ }^{3,4}$

It has been widely reported that microbioma modifications are associated to worse survival, and higher risk of infections and GvHD in patients undergoing allo-
HSCT $^{21,22}$ Therefore, efficacious decolonization strategies in this particular setting of patients are urgently needed.

Fecal microbiota transplantation is a fascinating decolonization strategy that has been proved to be efficacious in patients with recurrent $\mathrm{CDI} .^{23,24}$ On the other hand, concerns were initially raised for the use of FMT as a decolonization strategy in immunocompromised patients, due to the possible risk of local or systemic infections after the inoculum of microbiota pathogens.

Recently, DeFilipp et al. investigated the use of third-party FMT with the use of oral capsules as a strategy to restore microbioma diversity in patients undergoing allo-HSCT. The authors support the safety and feasibility of this procedure, underlining the possibility that microbiome restoration early after allo-HSCT may be of benefit. ${ }^{25}$

Here we describe the results of FMT in ten patients diagnosed with hematologic malignancies and undergoing FMT for MDRB colonization, namely CPE, CP-Pseudomonas aeruginosa or VRE, either before or after allo-HSCT. Decolonization was achieved in seven of ten patients, this being persistent at last follow up in six of ten patients. Our retrospective study not only suggests the efficacy of this procedure, but also its safety in patients with hematologic malignancies undergoing allo-HSCT. Interestingly, despite not being a selection criterion for FMT, we also registered patients concomitantly colonized from ESBL-producing enterobacteriaceae, with decolonization in three of six cases. We also showed that, in patients experiencing failure or relapse of MDRB colonization, a second FMT is feasible and efficacious. Interestingly, only three patients experienced significant infections after FMT. 
Moreover, it is worth underlining the significant benefit of major decolonization in the patient who had experienced multiple infectious episodes due to a CP-Pseudomonas aeruginosa, limiting breakthrough infections.

Our results also highlight that, despite the fact that administration of large spectrum antibiotics may hypothetically represent a risk for decolonization failure, the procedure remained effective in the majority of patients, without recurrence of $\mathrm{MDRB}$ in the majority of them despite use of broad spectrum antibiotics early after FMT. Interestingly, in one patient, VRE was detectable again at the time of disease relapse, despite no large-spectrum antibiotics having been used just before. One can speculate that disease relapse may probably have been associated to dysbiosis favoring selection of VRE, but conclusions cannot be drawn on just one case.

Despite the initial aforementioned concerns in immunocompromised patients, results of FMT in this setting are promising in terms of both efficacy and safety., ${ }^{4,16} \mathrm{~A}$ recent prospective study showed that FMT allowed total eradication of MDRB in $60 \%$ of cases, without any significant adverse event after the procedure. ${ }^{17}$ This is the only prospective study published to date using FMT in 20 patients with blood disorders and colonized with MDRB. Differently from our series, in this study, all types of MDRB were included and only a few patients underwent allo-HSCT.

In our Center, we only chose patients colonized with highly resistant bacteria, and in particular those classified as eXDR according to French guidelines or those known to cause a significant higher risk of systemic infection with very poor prognosis (i.e. CP-Pseudomonas aeruginosa).

So far, no specific guidelines have been defined as to the ideal timing, the best preparation of stools for FMT, and the best method of administration. In our experience, FMT was successfully undertaken either before or after allo-HSCT and, interestingly, it was also successful in two patients starting conditioning regimen for allo-HSCT three days after FMT.

As for stool preparation, frozen material was preferred in our center for logistical reasons, although in two cases fresh stools were used; this did not modify the results of FMT. It has recently been reported in a meta-analysis of patients receiving FMT for CDI, that the success rate of FMT was similar when using frozen or fresh stools. ${ }^{26}$ In contrast to most of the reported series of FMT for MDRB decolonization, we preferred enema as a method of administration as this is associated with lower risk of inhalation as compared to nasogastric administration.

The mechanisms underlying the efficacy of FMT for MDRB decolonization are still not clear. Recent studies showed that recipient stool assumed donor-like taxonomic and functional composition immediately following FMT. $^{27}$ Therefore, we hypothesize that FMT for MDRB decolonization works through the restoration of a more physiological microbiome, thus increasing the ecological pressure on MDRB. However, given the absence of translational studies on antibiotic resistance genes and microbiota composition on the patient's stool after FMT, we cannot exclude the possibility that FMT works through lowering MDRB below the threshold of detection rather than through true elimination.

In our series, after FMT, almost all patients had no major infectious complications during the first three months after FMT. Interestingly, in those patients subsequently undergoing allo-HSCT, no severe infectious bacterial complications occurred during the early transplant phase.

Regarding the impact of FMT on GvHD, only one of our patients had a grade IV acute gut GvHD concomitant to a carbapenemase-producing Pseudomonas aeruginosa at the time of FMT. In this specific case, the procedure was not efficacious either for MDRB or for GvHD. However, it is worth underlining that FMT was performed at a very late stage for compassionate use, and this may also explain the failure of the procedure. Importantly, among the nine remaining patients, only one experienced grade III acute gut GvHD after FMT (with a possible differential diagnosis with CMV colitis). A role for FMT in causing GvHD in this patient cannot formally be excluded and this point may be addressed in a prospective clinical trial.

Early studies in mice and humans suggested a link between gut microbiota and propensity to GvHD, with mice treated with gut-decontaminating antibiotics developing GvHD less often. ${ }^{28,29}$ Recent results of a pilot study also highlight the possible advantage of microbiota modulation with FMT in patients affected by steroid-refractory or steroid-dependent GvHD. ${ }^{30}$

With regards to donor choice, when available, people living in the same household of the patient were preferred as they widely share the same pathogens and environmental exposure, thus reducing the risk of transferring additional infectious agents from the donor to the recipient.

In line with previous reports, we consider that targeting gut microbiota in patients with impaired immune reconstitution in an attempt to reinstate a more equilibrated flora may favor stable eradication of the carrier status and prevent subsequent life-threatening infections.

This study has some limitations, including its retrospective nature, low number of patients, heterogeneous inclusion criteria, and differences in FMT procedure, making it difficult for any definitive conclusions to be drawn. However, we consider that our results support the use of FMT as a promising strategy to manage the considerable potential risks associated with the MDRB carrier status in immunocompromised patients with intestinal dysbiosis and in those patients having experienced single or multiple systemic infections. The majority of patients experienced no breakthrough infections after decolonization or $M D R B$ recurrence despite the use of broad spectrum antibiotics. Furthermore, our results provide fresh evidence of the safety of the procedure in this population, despite previous concerns in immunocompromised patients. These preliminary results underline the need for further prospective studies on the safety and efficacy of FMT.

\section{Acknowledgments}

The authors thank Prof. Junia V. Melo (University of Adelaide, Australia, and Imperial College, London) for medical editing of this manuscript.

\section{Funding}

This study was supported by educational grants from the "Association for Training, Education and Research in Hematology, Immunology and Transplantation" (ATERHIT, Nantes, France). 


\section{References}

1. Spellberg B, Guidos R, Gilbert D, et al. The Epidemic of Antibiotic-Resistant Infections: A Call to Action for the Medical Community from the Infectious Diseases Society of America. Clin Infect Dis. 2008;46(2):155-164.

2. Montassier E, Gastinne T, Vangay P, et al. Chemotherapy-driven dysbiosis in the intestinal microbiome. Aliment Pharmacol Ther. 2015;42(5):515-528.

3. Samet A, Śledzińska A, Krawczyk B, et al. Leukemia and risk of recurrent Escherichia coli bacteremia: Genotyping implicates E. Coli translocation from the colon to the bloodstream. Eur J Clin Microbiol Infect Dis. 2013;32(11):1393-1400.

4. Bilinski J, Robak K, Peric Z, et al. Impact of Gut Colonization by Antibiotic-Resistant Bacteria on the Outcomes of Allogeneic Hematopoietic Stem Cell Transplantation: A Retrospective, Single-Center Study. Biol Blood Marrow Transplant. 2016; 22(6):10871093.

5. Cattaneo C, Zappasodi P, Mancini V, et al. Emerging resistant bacteria strains in bloodstream infections of acute leukaemia patients: results of a prospective study by the Rete Ematologica Lombarda (Rel). Ann Hematol. 2016;95(12):1955-1963.

6. Birgand G, Leroy C, Nerome S, et al. Costs associated with implementation of a strict policy forcontrolling spread of highly resistant microorganisms in France. BMJ Open. 2016;6(1):1-9.

7. Lepelletier D, Berthelot P, Lucet J, et al. French recommendations for the prevention of "emerging extensively drug-resistant bacteria " (eXDR). J Hosp Infect. 2015;90(3):186195.

8. Bassetti M, Giacobbe DR, Giamarellou H, et al. Management of KPC-producing Klebsiella pneumoniae infections. Clin Microbiol Infect. 2018;24(2):133-144.

9. Tascini C, Sbrana F, Flammini S, et al. Oral gentamicin gut decontamination for prevention of KPC-producing Klebsiella pneumoniae infections: Relevance of concomitant systemic antibiotic therapy. Antimicrob Agents Chemother. 2014; 58(4):1972-1976.

10. Lübbert C, Faucheux S, Becker-Rux D, et al.
Rapid emergence of secondary resistance togentamicin and colistin following selective digestive decontamination in patients with KPC-2- producing Klebsiella pneumoniae: A single-centre experience. Int J Antimicrob Agents. 2013;42(6):565-570.

11. Rossen NG, MacDonald JK, De Vries EM, et al. Fecal microbiota transplantation as novel therapy in gastroenterology: A systematic review. World J Gastroenterol. 2015;21(17):5359- 5371.

12. Manges AR, Steiner TS, Wright AJ. Fecal microbiota transplantation for the intestinal decolonization of extensively antimicrobialresistant opportunistic pathogens: A review. Infect Dis (Lond). 2016;48(8):587-592.

13. Stalenhoef JE, Terveer EM, Knetsch CW, et al. Fecal microbiota transfer for multidrugresistant gram-negatives: A clinical success combined with microbiological failure. Open Forum Infect Dis. 2017;4(2):2-5.

14. Bilinski J, Grzesiowski P, Muszynski J, et al. Fecal microbiota transplantation inhibits multidrug-resistant gut pathogens: preliminary report performed in an immunocompromised host. Arch Immunol Ther Exp (Warsz). 2016;64(3):255-258.

15. de Castro CG Jr, Ganc AJ, Ganc RL, Petrolli MS, Hamerschlack N. Fecal microbiota transplant after hematopoietic SCT: report of a successful case. Bone Marrow Transplant. 2014;50(1):145.

16. Kelly CR, Ihunnah C, Fischer M, et al. Fecal Microbiota Transplant for Treatment of Clostridium diffi cile Infection in Immunocompromised Patients. Am J Gastroenterol. 2014;109(7):1065-1071.

17. Bilinski J, Grzesiowski P, Sorensen N, et al. Fecal microbiota transplantation in patients with blood disorders inhibits gut colonization with antibiotic-resistant bacteria: results of a prospective, single-center study. Clin Infect Dis. 2017; 65(3):364-370.

18. Magiorakos A, Srinivasan A, Carey RB, et al bacteria: an international expert proposal for interim standard definitions for acquired resistance. Clin Microbiol Infect. 2011;18(3):268- 281.

19. WHO. Antimicrobial resistance. Globa Report on Surveillance. Bull World Health Organ. 2014;61(3):383-394.

20. Schaik W Van. The human gut resistome.
Philos Trans R Soc Lond B Biol Sci. 2015;370(1670):20140087.

21. Taur Y, Jenq RR, Perales M, et al. The effects of intestinal tract bacterial diversity on mortality following allogeneic hematopoietic stem cell transplantation. Blood. 2017;124 (7):1174-1183

22. Jenq RR, Taur Y, Devlin SM, et al. Intestinal Blautia Is Associated with Reduced Death from Graft-versus-Host Disease. Biol Blood Marrow Transplant. 2015;21(8):1373-1383.

23. Austin M, Mellow M, Tierney WM. Fecal microbiota transplantation in the treatment of clostridium difficile infections. Am J Med. 2014;127(6):479-483.

24. Webb BJ, Brunner A, Ford CD, Gazdik MA Petersen FB. Fecal microbiota transplantation for recurrent Clostridium difficile infection in hematopoietic stem cell transplant recipients.Transpl Infect Dis. 2016;18(4): 628-633.

25. Defilipp Z, Peled JU, Li S, et al. Third-party fecal microbiota transplantation following allo-HCT reconstitutes microbiome diversity. Blood Adv. 2018;2(7):745-753.

26. Tang G, Yin W, Liu W. Is frozen fecal microbiota transplantation as effective as fresh fecal microbiota transplantation in patients with recurrent or refractory Clostridium difficile infection: A meta-analysis? Diagn Microbiol Infect Dis. 2017;88(4):322-329.

27. Moss EL, Falconer SB, Tkachenko E, et al Long-term taxonomic and functional divergence from donor bacterial strains following fecal microbiota transplantation in immunocompromised patients. PLos One. 2017;12 (8): $\mathrm{e} 0182585$

28. Jones JM, Wilson R, Bealmear PM. Mortality and gross pathology of secondary disease in 17 germfree mouse radiation chimeras. Radiat Res. 1971;45(3):577-588.

29. Shono Y, Docampo MD, Peled JU, et al Increased GVHD-related mortality with broadspectrum antibiotic use after allogeneic hematopoietic stem cell transplantation in human patients and mice Yusuke. Sci Trans Med. 2016:8(339):1-16

30. Kakihana K, Fujioka Y, Suda W, et al. Feca microbiota transplantation for patients with steroid-resistant acute graft-versus-host disease of the gut. Blood. 2016;128(16):20832088 